\title{
Francés para fines específicos en contexto politécnico
}

\author{
Ángeles LENCE \\ Universitat Politècnica de València \\ Departamento de Lingüística Aplicada \\ malence@idm.upv.es
}

Recibido: 22/07/2011

Aceptado: 06/02/2012

\begin{abstract}
Resumen
Desde finales de los noventa, el Francés para Fines Específicos (FOS) ha venido impartiéndose en la Universitat Politècnica de Valencia (UPV). Sin embargo, con la implantación de los nuevos planes de estudios, las asignaturas de lengua extranjera y en concreto de las asignaturas de francés, quedan relegadas a 4,5 créditos de libre elección, habiendo de competir, además, con la exigencia al alumnado del nivel B2 en lengua extranjera para poder graduarse. Teniendo en cuenta que el $95 \%$ del alumnado ha estudiado inglés en Secundaria y que, por lo tanto, es forzoso ofertar el nivel A1 de francés, ¿cómo afrontar la especificidad del francés para ingenieros? Esta y otras cuestiones vinculadas con la docencia, como la interculturalidad y el desarrollo humano sostenible, serán analizadas en este artículo con el objetivo de crear las mejores expectativas para el futuro del FOS en contexto politécnico.
\end{abstract}

Palabras clave: francés para fines específicos, ingenieros.

\section{Français sur objectifs spécifiques en milieu polytechnique}

\section{Résumé}

Vers la fin des années quatre-vingt-dix, on enseigne le Français sur Objectifs Spécifiques (FOS) à l'Université Polytechnique de Valencia (Universitat Politècnica de València-UPV-). Cependant, avec la mise en place des nouveaux plans d'études, les matières de langue étrangère et en particulier des matières de français, restent restreintes à 4.5 crédits de libre choix, devant exiger, de plus de la part des étudiants un niveau B2 en langue étrangère pour être diplômés. Tenant compte du fait que $95 \%$ des étudiants ont étudié l'anglais en Secondaire et qu'il faut donc offrir le niveau A1 de français, comment peut-on aborder, dans ces conditions, la spécificité du français pour ingénieurs? Cette question et d'autres liées à l'enseignement, comme l'interculturalité et le développement humain durable, seront analysés dans cet article dans le but de créer les meilleures perspectives pour le futur du FOS en milieu polytechnique.

Mots clés: français sur objectifs spécifiques, ingénieurs.

\section{French for Specific Purposes in Polytechnic Contexts}

\begin{abstract}
Since the late 1990s French for Specific Purposes (FSP) has been taught in the Universitat Politècnica de València (UPV). However, with the new curriculum, foreign languages courses, and in particular French, are not only given 4.5 elective credits, but they also compete with the fact that students must
\end{abstract}


achieve a B2 level in foreign languages in order to graduate. Taking into consideration that $95 \%$ of our students have studied English in secondary school and, consequently, they start studying French at an A1 level, how can we achieve the specific objectives in French for the Engineering degrees? This issue - and others related to teaching and learning, such as interculturality and sustainable developmentwill be analyzed in this paper with the aim of creating better prospects (?) for the future of FSP in Spanish polytechnic contexts.

Key words: french for specific purposes, engineering

\section{Referencia normalizada}

Lence, A. (2012) "Francés para fines específicos en contexto politécnico". Thélème, Vol. 27, 229-241.

Sumario: 1. Introducción. 1.1 Breve histórico del FOS en la UPV: francés en la ETSIT. 1.2 Contexto favorable: acuerdos con Escuelas homólogas francófonas. 1.3 Desaparición de Francés III: pérdida de FOS. 2. Actualidad de las asignaturas de Francés I y II en la ETSIT: proyectos colaborativos. 3. Cómo incorporar la especialidad en los niveles básicos de francés: uso de las TICs. 4. Futuro de la asignatura de Francés en las titulaciones de Grado en Ingeniería Técnica. 5. Conclusiones.

\section{Introducción}

\subsection{Breve histórico del FOS en la UPV: francés en la ETSIT}

Entre 1989 y 1997, al contrario de lo que sucedía en otras universidades, el FOS tomó un destacado impulso en la UPV y numerosas Escuelas ofertaron la asignatura de francés en sus Planes de Estudios, con una clara orientación a la formación de futuros ingenieros o arquitectos en su programa de enseñanza.

Pero no fue fácil en todas las Escuelas y fue la demanda de los estudiantes la que obligó a las autoridades rectorales a implantar su docencia. Es el caso de los estudiantes de la Escuela Técnica Superior de Ingenieros de Telecomunicación de Valencia (ETSIT) que sufrieron un duro trasiego durante varios cursos para asistir a las clases de francés. Al no tener su Escuela docencia asignada para impartirlas, se les brindó otra fórmula: acudir a la Escuela Técnica Superior de Arquitectura (ETSA) para asistir a las clases de Francés I y Francés II orientadas a la arquitectura ${ }^{1}$.

Sucedía entonces que el plan de estudios de la ETSA contemplaba las asignaturas de idiomas (Inglés y Francés) como obligatorias, lo cual quería decir que se les suponía ya a los estudiantes un nivel de lengua de COU o equivalente.

Estudiantes con este perfil eran, en su mayoría, los procedentes del Lycée Français, todos ellos bien preparados para abordar un texto técnico sobre arquitectura en el que descubrían un vocabulario específico que seguramente no necesitarían nunca pero que asumían con disciplina e interés.

\footnotetext{
${ }^{1} \mathrm{M}^{\mathrm{a}}$ Ángeles Lence: Proyecto Docente. Francés I. Francés II. Escuela Técnica Superior de Arquitectura, Universitat Politècnica de València, 1998.
} 
Fue en 1998 cuando las asignaturas de Francés I (Iniciación) y Francés II (Intermedio) se ofertaron en el entonces Nuevo Plan de Estudios de la ETSIT (Plan 96) con 6 créditos de libre elección para cada nivel. Para poder matricularse, los estudiantes debían estar cursando $4^{\circ}$ y $5^{\circ}$ de carrera. Se constituyeron dos grupos por asignatura, con una capacidad de 45 estudiantes por clase. No solamente se llenaban las aulas, sino que había una larga lista de espera.

En 2000 se ofertó una nueva asignatura, Francés III, son un solo grupo, orientada a fines específicos. Con 120 horas de francés cursadas, tratándose de alumnos y alumnas brillantes -la nota de corte para acceder a estos estudios era entonces muy alta- no era difícil alcanzar un nivel avanzado en esta asignatura.

\subsection{Contexto favorable: acuerdos con Escuelas homólogas francófonas}

Uno de los objetivos principales de la ETSIT ha sido siempre preparar bien a sus estudiantes para la empresa, teniendo en cuenta que los idiomas son imprescindibles para ser candidatos y candidatas a un buen puesto de trabajo, fuera y dentro de España. Con ese fin, a lo largo de los años esta Escuela ha desarrollado proyectos en colaboración con universidades de todo el mundo, firmando acuerdos de cooperación con Escuelas homólogas en Europa, en África y en América.

De manera especial y dentro del Programa Sócrates-Erasmus, destacan los acuerdos con las Escuelas homólogas francesas, siendo los más importantes los de doble titulación con Centros de gran prestigio como SUPELEC (École Supérieure d' Électricité) y ENSEA (École Nationale Supérieure de l'Électricité et de ses Applications) de París o TELECOMBretagne de Brest. Existen también acuerdos con las Escuelas de Lausanne, Lille, Bordeaux, Montpellier, Toulouse y otras Escuelas de París, para cursar 6 meses o un año completo.

Con el objetivo de beneficiarse de una beca Erasmus en país francófono, los estudiantes siempre han estado muy motivados para cursar las asignaturas de francés, pues saben que es requisito indispensable, no sólo para tener derecho a solicitar la beca, sino para poder asistir a clases de su especialidad en francés e incluso leer el Proyecto Fin de Carrera (PFC) en aquel país.

Los estudiantes que consiguen este Double Diplôme están muy bien considerados a nivel europeo por su alto nivel de preparación. Conseguir esta doble titulación significa también haber pasado un período de prácticas en una empresa francófona, uno de los requisitos -además de saber dos lenguas extranjeras- para obtener el título de ingeniero en Francia. Esto brinda también la posibilidad a nuestros estudiantes de encontrar más fácilmente un puesto de trabajo adecuado a su perfil en país francófono. Muchos de ellos trabajan actualmente en Francia o en países del Magreb, donde están bien considerados y muy bien remunerados si comparamos con las condiciones laborales del teleco en España. Y no porque en los últimos años España esté atravesando una de las peores crisis. Nuestros egresados siempre se han quejado del trato poco gratificante que han recibido en las entrevistas de trabajo en nuestro país y de los bajos sueldos en relación a la explotación que sufren cuando no tienen experiencia profesional. 
Por eso también muchos egresados prefieren dedicarse a la investigación y optan por las becas que oferta la propia universidad, sea para participar en un proyecto desarrollado por uno de los Institutos con sede en la Ciudad Politécnica de la Innovación o por un Departamento adscrito a la Escuela, por el Centro de Cooperación para el Desarrollo (CCD) o por cualquier otro organismo de la UPV. Durante este período pueden cursar el doctorado, optar por una plaza de profesor-ayudante, contratado, asociado-, doctorarse, acreditarse, hacer en fin carrera docente a la par que investigadora.

\subsection{Desaparición de Francés III: pérdida de FOS}

A la vista de este panorama podría deducirse que la asignatura de Francés III en la ETSIT gozaría durante años del apoyo académico. Así ha sido a lo largo de estos diez últimos años. Pero la UPV, desde su vocación de empresa -no olvidemos su contexto técnico, tecnológico e innovador- hace números y reparte el presupuesto anual a los Centros en función de las matrículas. Sujeto a este presupuesto y debido a la reducción de créditos por la disminución de matrículas -provocada por la saturación del mercado de las telecomunicaciones-, la ETSIT tiene en cuenta cada curso un mínimo número de matrículas en cada asignatura para formar grupos. Es así como su Plan de Ordenación Docente (POD) marca actualmente un mínimo de 10 matrículas para constituir grupo. Basándose en la matrícula del curso 2009/10, con 9 matrículas en Francés III, la asignatura dejó de ofertarse en el curso 2010/11.

Así pues, lo que fue todos estos años, el contexto del FOS y por ende, la salvaguarda del francés de especialidad -¿qué nos distingue si no de una academia de idiomas?- y la cantera de los potenciales Erasmus, ha sucumbido al presupuesto. No es suficiente el interés de nueve estudiantes por perfeccionar una lengua que les va a ayudar a abrir las puertas del mercado laboral extranjero, con sede dentro o fuera de nuestro país.

Sumada a esta pérdida para los estudiantes, la pérdida para la docencia y la investigación es enorme. El docente pasa a ser un especialista infrautilizado. La institución le convierte en un profesor de FLE cuyo trabajo bien podría hacerlo, por supuesto un profesor de francés de Secundaria, pero también cualquiera que haya obtenido el certificado de aptitud en francés en la Escuela Oficial de Idiomas. Lejos queda la época en que se le exigía al profesor universitario un Proyecto Docente orientado a los fines específicos que marcaba la Escuela o Facultad a cuya plaza optaba por oposición. ¿Para qué tantos años de dedicación y adaptación al contexto politécnico? Como filólogos, los profesores de francés en la UPV se han ido adaptando al medio, aprendiendo a utilizar los recursos que éste le ofrece, especialmente las llamadas nuevas tecnologías -ya no son tan nuevas- o tecnologías de la información y comunicación (TIC's) "com a alternativa o com a complement a les classes magistrals" (Lence, 2009: 440). Como filólogos, han compaginado su investigación, muchas veces literaria, con la lingüística aplicada que marcaban las necesidades del contexto. Y se han doctorado estos filólogos, teniendo siempre muy presente que su tesis había de constituir un puente entre los estudios puramente filológicos y los estrictamente técnicos. ¿Y para qué tanto esfuerzo? 


\section{Actualidad de las asignaturas de Francés I y II en la ETSIT: proyectos colaborativos}

Para responder a esta pregunta, se debe atender a las necesidades de los estudiantes y marcar unos objetivos a corto plazo. No hay que olvidar que son estudiantes de últimos cursos -y lo seguirán siendo en el Grado, tal como establece el plan de las nuevas titulaciones- y que están interesados en su mayoría por terminar sus estudios en país francófono.

El esfuerzo de un profesor e investigador del área de Filología Francesa en contexto técnico debe redundar en la formación lingüística, cultural y académicoprofesional del estudiante. En el aspecto lingüístico, es obvio que debe facilitar al discente la iniciación a la lengua francesa de modo progresivo y eficaz, puesto que dispone a lo sumo de 120 horas para que el principiante aprenda gradualmente a desenvolverse en francés. En el aspecto cultural, el docente ha de ayudar a descubrir al aprendiz una realidad diferente en costumbres y en el trato social, fuera y dentro del entorno académico. En el aspecto académico-profesional, el discente se enfrenta a otra manera de estudiar y de enfocar su futura profesión.

Y ahí radica la diferencia esencial entre un/a profesor/a de FOS y un/a profesor/a de FLE: la implicación de la docencia en FOS en medio universitario va desde el conocimiento general de los estudios de la Escuela en que se imparte y el conocimiento más concreto de las otras asignaturas con las que, en muchos casos, puede establecer relación e incluso coordinar acciones para un programa de aprendizaje "que enriquece a los profesores de diferentes áreas" (Lence \& Thome, 2008a: 293), hasta la familiaridad con los mecanismos que ayudan a los discentes a orientar su futuro profesional a través de la Oficina de Relaciones Externas o la Oficina de Relaciones con la Empresa que desde la propia Escuela informan sobre nuevos acuerdos, programas de movilidad y de intercambio, aulas de empresa, foros de empleo y un largo etcétera.

En la ETSIT el Área de Francés concede mucha importancia a los contactos con los Erasmus matriculados en sus cursos, una vez instalados en la ciudad e integrados en las Escuelas de destino de la beca. Son sin duda transmisores del nuevo entorno y de otra realidad. No sólo transmiten lo que en materia educativa está aconteciendo en ese contexto, sino también lo relativo a su cultura "pour mieux apprendre l'autre" (LeSaout, 2008: 69).

Esta interrelación se extiende además a Francia con cuyas universidades colaboramos desde hace dos décadas para realizar proyectos pedagógicos que se concretan a su término con viajes de estudios en los que la reciprocidad es esencial, permitiendo "un dinamismo mayor que el que es posible conseguir en la clase tradicional" (Lence \& Thome, 2008b).

En el curso 2010/11, por ejemplo, se inició Francés I en el primer cuatrimestre con un objetivo añadido a los objetivos del curso: una visita de intercambio a TELECOMLille1, donde, en coordinación con profesores y grupos de español de esa Escuela, los grupos de Francés I, con su profesora, pasaron una semana de inmer- 
sión lingüística, cultural y académica. El programa de enseñanza impartido por la profesora de francés, dentro del Programa Sócrates-Erasmus, fue el siguiente:

MAÑANA / MATIN

$2 \mathrm{~h}$ diarias de clase / $2 \mathrm{~h}$ de cours par jour

$2 \mathrm{~h}$ examen en línea / $2 \mathrm{~h}$ examen en ligne

$\rightarrow$ certificado nivel Bulats on line ${ }^{2}$

$\rightarrow$ attestation niveau Bulats en ligne

\section{TARDE / APRÈS-MIDI}

Actividades / Activités

-ir al supermercado / aller au supermarché

-ir a la Oficina de Turismo / aller à l'Office de Tourisme

-ir a un espectáculo / aller à un spectacle

-ir a un museo/una exposición / aller à un musée/une exposition

-ir a una librería / aller à une librairie

-fiesta española / soirée espagnole

$\rightarrow$ INFORME/compte-rendu

Los objetivos de la movilidad y los valores añadidos de la movilidad (tanto para la institución de destino, como para los visitantes) son:

-El intercambio lingüístico-cultural y académico-profesional entre estudiantes españoles y estudiantes franceses de Telecomunicaciones que estudian francés y español respectivamente

- Las prácticas de francés con objetivos específicos para el futuro ingeniero.

Los resultados esperados y conseguidos son:

- La inmersión sin barreras lingüísticas de nuestros estudiantes en situaciones de la vida real

—La relación académica y personal entre estudiantes homólogos que motiven la movilidad interuniversitaria

- La preparación de nuestros estudiantes para el perfil de ingeniero en empresa francófona (obtención del certificado de nivel BULATS).

Además, se programaron dos horas diarias de seminario impartidas por el profesor acompañante, responsable de la Subdirección de Calidad de la ETSIT. Estos

\footnotetext{
${ }^{2}$ El examen en línea BULATS (Business Language Testing Service) evalúa las competencias lingüísticas en general y las relacionadas con los negocios.
} 
seminarios, dirigidos a profesores y estudiantes de español de TELECOMLille1 incluyen los siguientes temas:

-Presentación de la UPV/Présentation de l'UPV

- Presentación de la ETSIT/Présentation de l'ETSIT

-Entorno socio-económico de Valencia/Contexte socio-économique de Valencia

- Comparativa con TELECOMLille1/Analyse contrastive ETSITTELECOMLille1

-Perspectivas de colaboración/Projets de collaboration

- Como objetivos, aparte de los ya indicados más arriba, se marcaron los siguientes:

-Presentación y análisis de nuestras respectivas Escuelas para desarrollar una colaboración continua

- Suscitar un debate entre estudiantes sobre las similitudes y diferencias de ambas Escuelas con el objetivo de regular un acuerdo de colaboración

Hay que señalar que, en el momento de realizar esta estancia, los estudiantes de Francés I estaban cursando Francés II, de modo que su nivel había progresado. Una vez en Lille, recibieron clases de preparación al Test Bulats que, coordinado desde los Cursos Internacionales de la Universidad de Salamanca, genera un examen de francés en línea enfocado a objetivos específicos relativos al campo de la empresa y en el que el nivel lo va estableciendo el propio alumno a través de sus respuestas. Siguiendo una progresión en la dificultad de los ejercicios, el test otorga un nivel al alumno de acorde al Marco Común Europeo de Referencia para las Lenguas ${ }^{3}$ en las siguientes competencias:

\section{Comprensión de Lectura}

Comprensión Auditiva

Conocimiento de la Lengua (Gramática y Vocabulario)

De esta forma, se posibilita al estudiante la obtención de un certificado de nivel que actualmente es requisito para solicitar una beca Erasmus y que, además, será un valor añadido en su currículum a tener en cuenta en cualquier baremación de méritos.

Aunque no pueden compararse 10 horas de clase con las 60 horas que comprendía la asignatura de Francés III, este curso intensivo en Lille viene a compensar mínimamente el dossier asociado a esa asignatura sobre cómo prepararse para una

\footnotetext{
${ }^{3}$ Common European Framework of Reference for Languages: Learning, Teaching, Assessment. Strasbourg, Council of Europe, 2001.
} 
candidatura a un puesto de trabajo en empresa francófona: redacción de cartas de presentación respondiendo a una oferta de trabajo, elaboración del currículum, preparación para una entrevista telefónica o presencial..., contenidos que los egresados han mencionado haberles sido muy útiles al finalizar los estudios.

Cabe señalar también que el grupo de español de TELECOMLille1 visitó después la ETSIT, aplicando en su caso un programa de enseñanza similar. Para tal fin, la Escuela receptora solo ha de tener disponible un aula convencional para las clases diarias y un aula de informática para la realización del examen en línea. La recepción del grupo por parte del director de la Escuela, la acogida de los estudiantes a los visitantes, la realización de un programa lúdico, un itinerario turístico, son actividades que fortalecen las relaciones entre las Escuelas.

\section{Cómo incorporar la especialidad en los niveles básicos de francés: uso de las TICs}

Se ha expuesto una actividad que palia en parte la desaparición de la única asignatura que estaba orientada a fines específicos. Para no ser un simple Centro de Lenguas ni una Academia de Idiomas, los profesores universitarios de francés han de distinguirse por los objetivos específicos de su programa de enseñanza. ¿Es esto posible cuando se empieza en la lengua desde cero?

Es evidente que hay que centrarse primero en la fonética y en la gramática para llegar a este enfoque. Que el ritmo de aprendizaje es diferente en cada individuo y consecuentemente el progreso de la lengua en cada estudiante. No obstante, se puede utilizar material complementario al libro del alumno, procurando que ese material sea relativo a su especialidad: artículos de prensa sobre telecomunicaciones, arquitectura, agronomía o cualquier otro dominio técnico, webs científico-técnicas francófonas, presentaciones.

Durante el curso pasado, por ejemplo, una estudiante Erasmus en la École Polytechnique Fédérale de Lausanne (EPFL), hubo de examinarse de Francés I a distancia. Se le propuso un tema: Lausanne, desde el punto de vista cultural, lingüístico, académico -su Escuela- y profesional -marco laboral y posibilidades de empleo-. En diez transparencias elaboró un documento visual y descriptivo en francés. Tras su revisión y corrección y previa autorización de la estudiante, se presentó en clase. Los estudiantes recibieron la consigna de formular preguntas a partir del documento. Estas preguntas se trasladaron a la estudiante cuya respuesta grabó y envió en un fichero que los estudiantes escucharon en otra sesión.

El trabajo colaborativo se implementa con el uso de las nuevas tecnologías que aportan "materiales de estudio digitales que podrán ser utilizados por otros profesores y estudiantes; promoviendo de esta manera, la conformación de redes de aprendizaje" (Enríquez \& Chaos, 2006). En efecto, la utilización de metodologías activas permite trabajar con grupos de Español Lengua Extranjera (ELE), haciendo prácticas síncronas o asíncronas. Como prácticas síncronas se ha puesto en marcha desde hace al menos una década, la utilización del chat y de la conversación telefónica 
(dialogues en ligne) con resultados diversos, en los que la "immediatité du dialogue en ligne rend plus facile et plus fréquent l'échange entre étudiants" (Lence, 2009b: 250). Como señala el profesor LeSaout, estas prácticas forman parte de una "pédagogie assistée par les multimédias et à la poursuite de la recherche dans le domaine de l'Espace Interculturel" (2008: 63). Excelentemente equipadas las salas informáticas de la ETSIT y las Escuelas homólogas, los docentes de uno y otro centro coordinan estos intercambios desde el comienzo de curso, teniendo en cuenta el nivel de los estudiantes y el horario de cada asignatura, de forma que coincidan en tiempo real dos grupos de nivel similar. Cada estudiante tiene un corresponsal asignado al azar para todas las sesiones.

Basado en la reciprocidad, el intercambio consiste en compartir cada sesión alrededor de un tema, distribuyendo el tiempo de forma equitativa para las dos lenguas en juego, así como en la corrección entre pares.

SKYPE es la herramienta que mejor ha funcionando para realizar estas prácticas. Se ha alternado el uso del chat -modo en el que los estudiantes se encuentran más cómodos- y de la conversación telefónica. La herramienta posibilita además el envío de documentos, enlaces, imágenes... que enriquecen los diálogos. El chat ofrece la posibilidad de guardar las conversaciones, de modo que, al acabar las sesiones, los estudiantes las envían a sus respectivos profesores para su revisión y posterior puesta en común en el aula.

Como práctica asíncrona, combinada o no con la síncrona, el correo electrónico es utilizado entre los estudiantes de forma más autónoma y menos dirigida, siendo los temas de conversación a elección de los estudiantes. A lo largo del curso deben redactar un informe que recoge los diversos asuntos que han tratado para su posterior revisión por el docente y exposición en el aula por el estudiante: "c'est dans ce rapport que tous les mécanismes de la créativité se mettent en marche" (Lence, 2002: 105).

Y ya de forma voluntaria y particular, los estudiantes de ambas Escuelas se han ido agregando como amigos en Facebook y redes sociales similares, extendiéndose en consecuencia sus contactos. Desde el punto de vista cultural, este fenómeno es muy importante, porque para un estudiante español se le abre la posibilidad de comunicarse con todo el mundo francófono y para el estudiante francés el amplio panorama hispanófono. Según Juan "el medio solo propicia que el estudiante o el profesor puedan seleccionar aquellos formatos con los que se sientan más cómodos para "comunicar" de forma más efectiva un conocimiento o una información" (2009: 6-8).

Los excelentes resultados de estas prácticas motivan a seguir innovando la metodología en el aula de francés, estando en preparación para su inmediata puesta en marcha una experiencia piloto de aula virtual, inspirada en el programa Escuela 2.0 ${ }^{4}$.

\footnotetext{
${ }^{4}$ El Programa Escuela 2.0 es un proyecto de integración de las TICs en los centros educativos, que contempla el uso personalizado de un ordenador portátil por parte de cada alumno o alumna. El
} 
La UPV dispone ya de varias aulas virtuales que, más allá de la videoconferencia, puede conectar grupos de enseñanza a distancia. Si la videoconferencia limita a sus participantes a verse y hablar sin posibilidad de intercambiarse documentación o siendo ésta muy engorrosa - utilizando un ordenador paralelamente-, el aula virtual, compuesta por una pizarra digital interactiva, cañón proyector, un ordenador portátil para cada participante y varias webcams distribuidas por el aula, permite comunicarse, verse, utilizar todos los recursos al alcance -material multimedia, videos, presentaciones en PowerPoint...- incluso dar una clase ${ }^{5}$, a través de Polireunion ${ }^{6}$.

Otra posibilidad es la utilización del espacio virtual. La diferencia respecto al aula virtual es que permite la conectividad fuera de ella. Sólo se necesita que cada corresponsal tenga un ordenador portátil y reserve ese espacio en la aplicación correspondiente. La UPV ha diseñado esta aplicación -Policonecta- y actualmente se utiliza para tutorías a distancia ${ }^{7}$.

Todas estas herramientas motivan a los estudiantes politécnicos al aprendizaje del idioma y se debe hacer un esfuerzo por utilizarlas en el aula. La UPV organiza con frecuencia cursos de formación donde se aprende su manejo, aunque siempre sea recomendable el asesoramiento de un técnico que vele por el buen mantenimiento de los recursos tecnológicos y de los problemas técnicos que puedan surgir. Asimismo los propios estudiantes, inmersos en este contexto digital, ayudan a los docentes a optimizar estos recursos en muchas ocasiones.

Esta cultura digital es también el nuevo contexto de la sociedad y tiene efectos en la ciudadanía, en su entorno urbano, en la administración, en las relaciones -no hay que olvidar el gran auge que han tomado las redes sociales- en la creatividad, en el mundo de la empresa, en la innovación, en el emprendedurismo..., pero sobre todo en la educación y en el aprendizaje. A esta forma de "interacción que combina lo social y lo tecnológico se la llama Cyborg (homo digitalis), es decir la extensión de las capacidades humanas a través de la utilización de las TIC" (Cobo, 2007: 5). Los ingenieros del futuro, en la actualidad estudiantes, deben tomar conciencia de esta revolución creativa y social, de forma que sean capaces de construir una nueva sociedad, más cohesionada y más colaborativa entre los seres humanos, sin olvidar que existe una brecha digital por superar. En este punto, el Desarrollo Humano Sostenible está marcando una pauta de trabajo en la UPV. Desde el Centro de

objetivo supone poner en marcha las aulas digitales del siglo XXI, aulas dotadas de infraestructura tecnológica y de conectividad. Instituto de Tecnologías Educativas Ministerio de Educación http://www.ite.educacion.es/

${ }^{5}$ Para compartir fácilmente los archivos de proyectos, colaborar en documentos y organizar conferencias web, contamos con el programa https://acrobat.com/welcome.html

${ }^{6}$ Polireunion permite agilizar, controlar y cuantificar el uso de las nuevas salas virtuales disponibles para el personal de la Universitat Politècnica de València: https://polireunion.upv.es

${ }^{7}$ Policonecta permite compartir desde el ordenador del profesor aplicaciones con los alumnos: http://cfp.blogs.upv.es/tag/policonecta 
Cooperación para el Desarrollo (CCD), se lanzan programas de cooperación que intentan hacer más pequeña esta brecha. Muchos de los países con los que la UPV tiene firmados acuerdos de cooperación pertenecen al mundo francófono -Burkina Faso, Côte d'Ivoire, países del Magreb...- y los estudiantes politécnicos son potenciales candidatos a cubrir las plazas que se ofertan con el requisito de tener un buen nivel de francés.

En estos momentos la ETSIT tiene egresados repartidos por el mundo. Son antiguos estudiantes que un día se beneficiaron de una beca ERASMUS en Francia o país francófono, hicieron allí prácticas en empresa, obtuvieron su doble titulación, encontraron un buen puesto de trabajo en empresas del sector de las telecomunicaciones y actualmente están satisfechos con su situación laboral, con la buena consideración que de ellos tienen sus superiores y una excelente remuneración. O son estudiantes que decidieron hacer su Proyecto Fin de Carrera (PFC) en temas de cooperación y hoy en día llevan proyectos solidarios en países subdesarrollados o emergentes. Es el caso de estudiantes que han montado una radio local en Burkina Faso o que han diseñado redes locales que interconectan centros de salud en lugares remotos y de difícil acceso.

\section{Futuro de la asignatura de Francés en las titulaciones de Grado en Ingenie- ría Técnica}

Para la Universitat Politècnica de València y para todas las universidades debe ser imprescindible poner en valor los recursos docentes y humanos de las lenguas extranjeras, ya que esto supone empleabilidad, convergencia y movilidad de nuestros egresados, así como una herramienta imprescindible en la investigación. Por ello se hace especial énfasis en mantener una propuesta potente en inglés, pero es necesario completarla con Francés y Alemán, pues la formación multilingüe es un valor de presente y futuro.

De acuerdo con lo indicado en el punto 8 del Documento Marco para el diseño de titulaciones ${ }^{8}$, aprobado por Consejo de Gobierno el 14 de febrero de 2008, en relación con el tratamiento de las lenguas extranjeras, para facilitar el aprendizaje requerido, la planificación de las enseñanzas en las titulaciones de Grado de la UPV incorporará, al menos, una asignatura de 4,5 créditos ECTS. Estas asignaturas, excepcionalmente, se organizarán por el Departamento de Lingüística Aplicada, con una docencia, al menos, de 90 horas presenciales.

\footnotetext{
${ }^{8}$ Diseño de titulaciones: Documento Marco. Vicerrectorado de Estudios y Convergencia Europea, UPV, 2008, disponible en: http://www.etsia.upv.es/NuevosPlanesEstudio/Documentos/Dise\%C3\% B10\%20Titulaciones_UPV.pdf
} 
Y hay que destacar que en la página 23 de ese mismo Documento Marco, en el capítulo 8 -"Lenguas extranjeras y otras competencias transversales"-se establece que "(sic) para la obtención del título de Grado, el alumno deberá acreditar el conocimiento de una lengua extranjera, preferentemente el inglés, con un nivel correspondiente al B2 de los niveles comunes de referencia fijados por el "Marco común europeo de referencia para las lenguas: aprendizaje, enseñanza, evaluación".

Cabe la posibilidad de que algunos estudiantes opten por la nueva asignatura de Francés equivalente a 4,5 créditos ECTS.

Sin embargo, en la reciente distribución del POD para el curso 2012-13, por decisión unilateral del Vicerrectorado del Profesorado y Ordenación Académica, se ha incumplido con lo dispuest en el Documento Marco; es así como no se ha tenido en cuenta la duplicidad de créditos en Francés y Alemán cuyo nivel B2 equivladrá, al menos en el próximo curso, a 45 horas presenciales. Únicamente Inglés B2 se beneficiará de la duplicidad de créditos. Decisión que, así se nos ha trasmitido, será revisable en un futuro.

El reto está, por una parte, en conseguir que esos estudiantes puedan alcanzar el nivel B2 que se les exige para la titulación de Grado, por otra que esa asignatura pueda orientarse al FOS.

\section{Conclusiones}

Desde los años noventa, las asignaturas de Francés de la ETSIT han formado parte de programas de Innovación Educativa promovidos por la propia Universidad, con el fin de mejorar la metodología docente y de motivar a los estudiantes con acciones participativas. Para conseguir estos objetivos, se ha contado con la complicidad de profesores de diferentes materias técnicas que, conocedores de las necesidades académicas y profesionales de cada sector, asesoran a los profesores de lengua extranjera en lo relativo al perfil y desarrollo personal y profesional del futuro ingeniero. Con este tándem formado por el lingüista y el ingeniero se consigue el acercamiento a la filosofia del EEES al conciliar el savoir faire con el savoir être de los estudiantes.

Ahora bien, desde la docencia, no se pueden cambiar las normativas aprobadas en los órganos colegiados de las respectivas universidades que parecen confrontar estos valores. La convergencia europea ha trazado una línea a seguir a la que se han ido adaptando las nuevas titulaciones de toda la universidad española. En la UPV la nueva estructuración de las carreras deja un margen bastante pequeño a las lenguas extranjeras, situando el inglés por encima de las demás -con el argumento de que a los bachilleres se les supone un nivel B1 en esta lengua- y poniendo en peligro la especialidad en la segunda lengua extranjera, sea Francés o Alemán.

Desde la docencia, sí se puede cambiar, aún más si cabe, las estrategias de aprendizaje en el aula, aprovechando todos los recursos que el entorno politécnico nos ofrece, aprendiendo a utilizarlos para su mayor optimización didáctica. Si a los recursos técnicos y tecnológicos, sumamos los recursos humanos disponibles, 
gracias a los acuerdos firmados por las Escuelas de Ingenieros con sus homólogos europeos o iberoamericanos, coordinando proyectos de colaboración que redunden en la participación de los estudiantes en programas de intercambio, se puede asegurar la permanencia del FOS en las aulas.

\section{REFERENCIAS BIBLIOGRÁFICAS}

Cobo, C., (2006) "Las multitudes inteligentes de la era digital" in Revista Digital Universitaria [En línea]. Vol. 7, n⿳0 6 , junio 2006, pp. 1-17, disponible en: http://www.revista.unam.mx/vol.7/num6/art48/int48.htm [Último acceso el 20 de febrero de 2012].

Enríquez, L. \& L. Chaos, (2006) “Movilidad y educación: m-learning” in Enter@te en línea [En línea]. Año 5, n⿳5口, noviembre 2006, disponible en: http://www.enterate.unam.mx/Articulos/2006/noviembre/m-learning.htm [Último acceso el 23 de febrero de 2012].

Juan, O., (2009) "Redes sociales, Web 2.0 y el español: estrategias y recursos. Anotaciones en torno a la conferencia en el XVIII Encuentro Práctico, Barcelona". Disponible en: http://www.encuentro-practico.com/pdf09/juan-web.pdf [Último acceso el 23 de febrero de 2012].

Lence, M.A., (2002) "Langue et interculturalité: échanges par courriel entre deux écoles des télécommunications" in Langues spécialisées et besoins spécifiques: théorie et pratique. Evry, Actas de GLAT, pp. 101-110.

Lence, M.A., (2009a) "Implantació de metodologies actives en l'aula universitària: les TIC en l'aprenentatge del francès" in I Congrés internacional d'Interacció comunicativa i ensenyament de llengües. Valencia, Universitat de València, pp. 439-445.

Lence, A., (2009b) "Nouvelles technologies et apprentissage du français en milieu universitaire: dialogues entre étudiants en télécommunications" in Synergies Espagne, revue $d u$ GERFLINT [En línea]. $\mathrm{N}^{\mathrm{o}} 2$, pp. 245-250, disponible en: http://ressources-cla.univ-fcomte.fr/gerflint/Espagne2/lence.pdf [Último acceso el 23 de febrero de 2012].

Lence, M.A. \& N. Thome, (2008a) "Algunas actividades docentes llevadas a cabo en la Universitat Politècnica de València de España" in Actas de la II REPEM. Santa Rosa, Universidad Nacional de La Pampa, pp. 288-294.

Lence, M.A. \& N. Thome, (2008b) "Experiencia piloto de intercambio lingüísticocultural entre estudiantes de Telecomunicaciones de una universidad española y de otra francesa" in Actas del XVI Congreso Universitario de Innovación Educativa en las Enseñanzas Técnicas. Cádiz, Universidad de Cádiz, pp. 2-10.

LeSaout, A., (2008) "Intégration de la nouvelle technologie dans le domaine de la linguistique interculturelle" in Enseignement des langues et formation interculturelle. Quels enjeux pour les entreprises, les écoles d'Ingénieur et de Management. Paris, UPLEGESS, pp. 62-71. 